\title{
Hydatid Cyst in Parotid Gland- A Rare Case
}

\author{
Dr D B Singh*, Dr Sunil kumar**, Dr Aarti B.Bhattacharya*** \\ *Assistant Prof. Dept. of ENT \& HNS Hind Institute of Medical Sciences Safedabad Barabanki UP India \\ **Assistant Prof. Dept. of ENT \& HNS K.G.M.U. Lucknow UP India \\ ***Associate Prof. Dept. of Pathology Hind Institute of Medical Sciences Safedabad Barabanki UP India
}

\begin{abstract}
Hydatid disease in the maxillo-facial region accounts for only $2.0 \%$ as compared to liver (60-75\%) and lungs $(15-25 \%)$. Here in, we report a rare case of hydatid disease in the right parotid gland in a young female from a rural farming background. A detailed history including occupational history gives clue to Hydatid disease as a differential diagnosis. Patients of hydatid disease must undergo a thorough systemic investigation as $20-30 \%$ patient's have multiorgan involvement. In the present case only right parotid was involved. Thus, in non endemic areas, ecchinococcosis of head and neck is very rare and therefore its conscious inclusion in the differential diagnosis helps.

Key Words: Primary, infratemporal swelling, Echinococcosis, Hydatid cyst, Parotid Swelling.
\end{abstract}

\section{Introduction}

Hydatid disease is caused by the cestodes of Echinococcus granulosus $(1,3)$.The parasite has dog- sheep cycle, but man becomes an accidental intermediate host. The most commonly involved organs are liver(60-75\%) and lungs(15-25\%). Any organ or tissue can be the primary site of hydatid disease. (4) Hydatid cysts are very rarely seen in head and neck region. Very few cases of hydatid cyst in infratemporal region have been reported in literature(3). We report an unusual case of hydatid cyst in the right infraauricular region(Right Parotid gland). This is a reminder for the clinicians that for a slow growing benign cystic lesion in any region, hydatid cyst should be included in differential diagnosis.

\section{Case Report:-}

History: A 35 year old female from rural Eastern UP had presented with a slowly progressive five year swelling in the right infra auricular region. She did not complain of pain or pressure sensation. Patient's occupation was agriculture and she gave history of close association with cow, buffalo, sheep and dog.

Clinical features: On examination there was a firm, nontender swelling in the right infra auricular region. General health of the patient was otherwise normal. There was no cervical lymphadenopathy, chest radiograph, blood and urine analysis were normal except for a slight increase in eosinophil count.

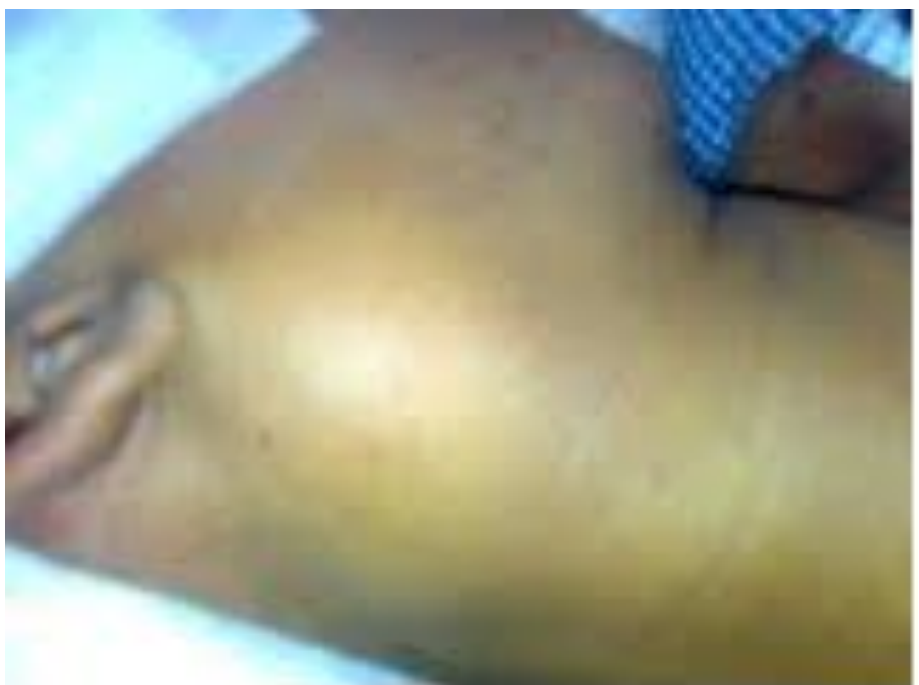

Width (Px): 161, Height (Px): 121 Color Depth:
Firm cystic swelling in the right infra auricular region.

Figure-1 (a) 


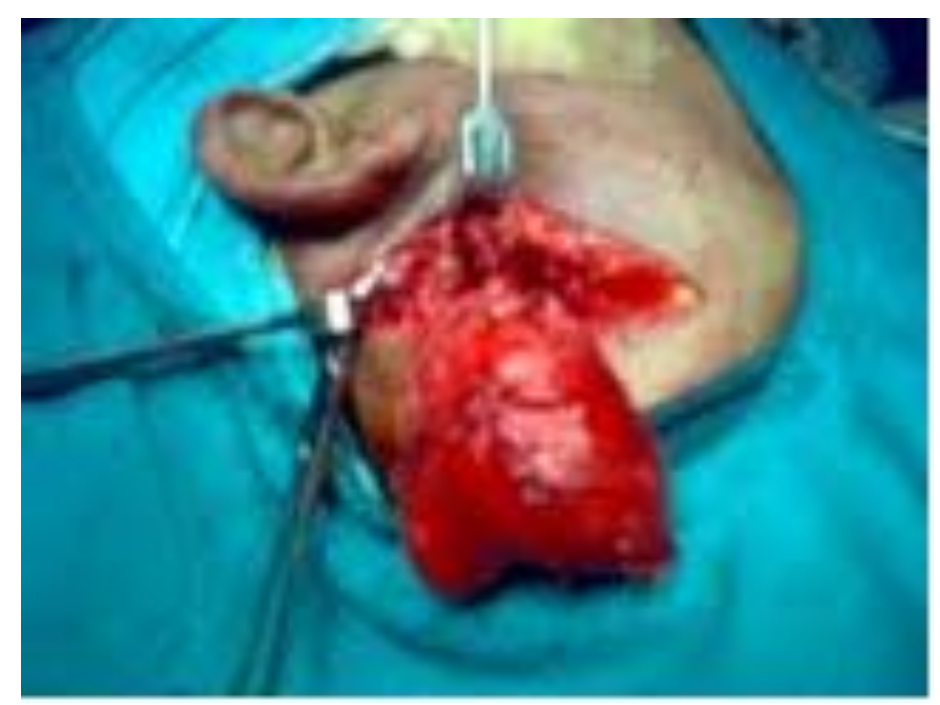

Per-op findings of Hydatid cyst.

Figure-1 (b)

Width (Px): 161, Height (Px): 121

Color Depth:

Ultrasonography: revealed a well encapsulated multiseptate cystic mass in the right infra auricular region. Parotid gland could not be discerned seperately.

\section{FNAC diagnosis:- Lymphoepithelial cyst.}

The benign cystic lesion was excised in totto. No difficulty was encountered during the surgery. Recovery was uneventful.

\section{Gross Pathology:}

The cyst measured $6 \times 3.5 \times 3 \mathrm{~cm}$. External wall was whitish and refractile. Cut surface revealed a multiloculated cyst with scanty serous fluid.

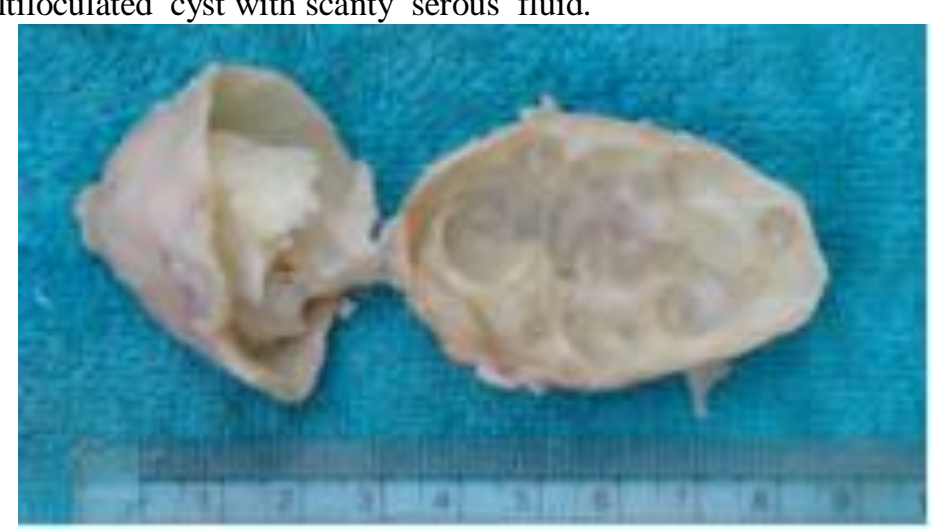

Cut surface of Hydatid Cyst.

Figure-1 (c)

Width (Px): 212, Height (Px): 118

Color Depth:

\section{Histopathology:}

H\&E stain revealed the germinal layer(endocyst), avascular eosinophilic refractile chitinous layer (exocyst)and the outermost adventitial layer(pericyst). On the outer aspect of this layer mucinous acini of parotid were seen. A diagnosis of Hydatid cyst was given. 


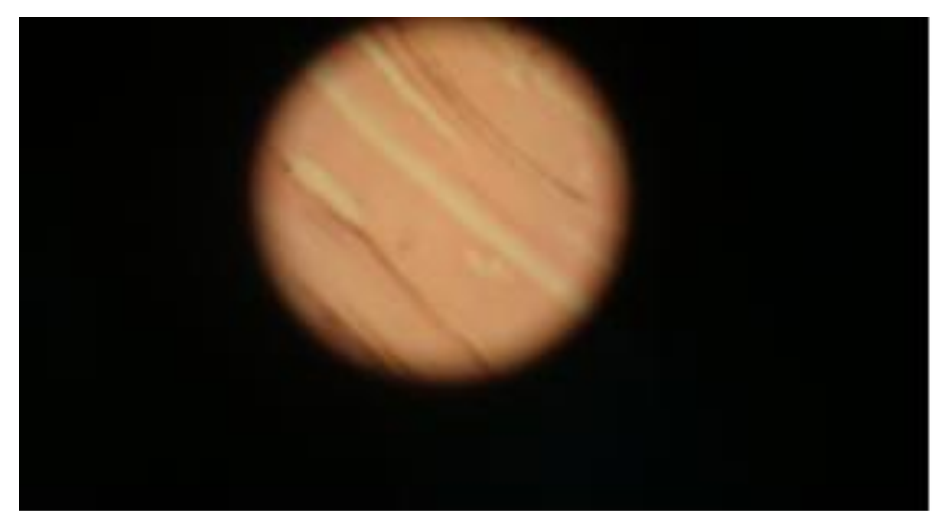

Microscopic view of Chitinous layer of Hydatid Cyst (H\&Ex40)

Figure-1 (d)

Width (Px): 280, Height (Px): 154

Color Depth:

JOMFP_333_1114.jpg

Click on image to view in full size

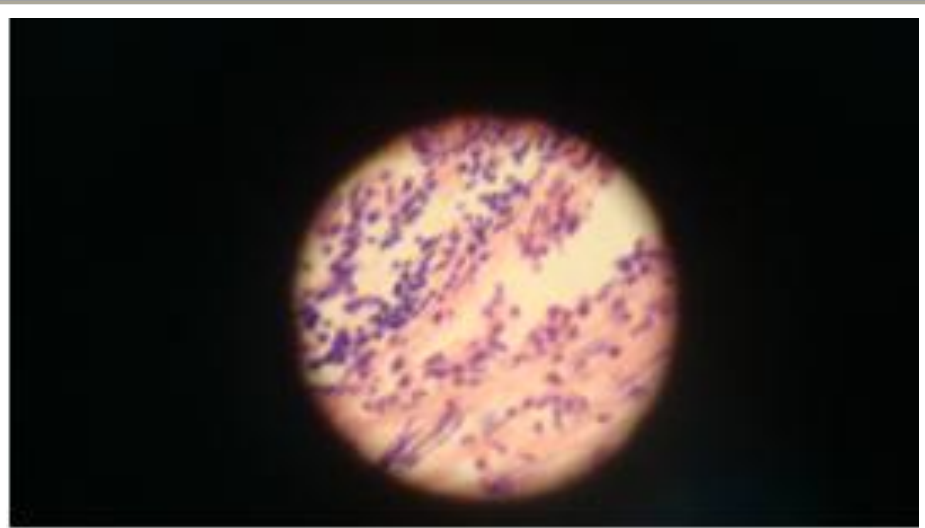

Microscopic view of Pericyst of

Hydatid Cyst (H\&Ex40)

Figure-1 (e

Width (Px): 270, Height (Px): 152

Color Depth:

Patient received albendazole $50 \mathrm{mg} / \mathrm{Kg} /$ day for 2 years .Patient has been disease free since 2 years.

\section{Discussion:-}

Hydatid cyst in the maxillofacial region are rare accounting for only about $2 \%$ of hydatid infections of the body. Hydatid cysts in the maxillofacial region are not usually considered in the differential diagnosis of head and neck cystic swelling especially in nonendemic areas and in the absence of hydatid disease elsewhere in the body. Humans are accidental host and do not play a role in the biological cycle of Ecchinococcus Granulosus[3]. E granulosus, a small (3-5mmlong) tapeworm resides in the jejunum of $\operatorname{dog}$ (definitive host) and other canines and produces eggs that are trapped in stool. Definitive host may be infected with thousands of worms. These worms produce eggs that are released to environment and are infective to intermediate hosts like sheep, and accidentally, to humans. Once the eggs are ingested with contaminated fruits or vegetables, digestive enzyme liberates an embryo in duodenum that passes through intestinal mucosa to portal circulation and migrates to visceral organs. As liver filters out most of the larvae, most cysts occur in the liver [3]. Later on fluid filled cyst develops, that differentiates into three layers to form Hydatid cysts [6]. The wall of the cyst is composed of outer layer formed by host, inner two are formed by parasite. The inner most parasitic lining gives rise to 'hydatid sands' which are brood capsules or daughter cysts in which scolices are formed [5]. Of the reported cases, 3 involved the tongue, 2 involved the cheek and very few involved the infratemporal fossa [7]. Hydatid disease is more prevalent in individuals living under poor socioeconomic conditions and have a poor health status[5,7]. Georgopaulus et al for the first time reported a hydatid cyst in submandibular gland. It has been observed that a solitary organ involvement without evidence of hepatic or lung involvement occurs eventhough embryos must have passed through the organs (3). According to literature rural women and children are more closely associated with domestic farming duties and hence are more susceptible as in our case. Prevalence is highest in second to fourth 
decades of life, even though humans usually become affected during childhood. It has been observed that the interval between the initial infection and clinical symptoms is variable. These lesions are characteristically slow growing and well tolerated and the manifestation of symptoms depend on the location, size and pressure caused by enlarging cyst.[5,8]. Past medical history, family history, patient's occupational history and residence may suggest diagnosis of hydatid cyst in differential diagnosis. Unless suspected or demonstrated (Radiology) preoperative diagnosis may be missed [8,9]. Fine needle aspiration cytology is efficient in diagnosing hydatid cyst in soft tissues but usually it is avoided as it can precipitate acute anaphylaxis and also spread daughter cysts $[4,8]$.

Our fine needle aspiration cytology report was Lymphoepithelial cyst. Hydatid cyst was diagnosed post operation on gross examination and microscopy. Diagnostic imaging has been greatly facilitated with Ultrasonography, CT and MRI. Serological tests like. ELISA, Latex agglutination and Indirect Haemagglutination tests have low sensitivity and low specificity. The importance of serologic tests lies in the follow-up of treated patient in whom a drop in titer indicates resolution and a rise indicates recurrence of cysts. Surgery is the mainstay of treatment. When diagnosed before surgery, inactivation of daughter cysts and scolices is done by injecting $20 \%$ hypertonic saline or 5\% silver nitrate into the cyst [2]. Combination of medical therapy with imidazole derivatives has been used for the management of patients with recurrence and high risk contamination [1,2,8]. Our patient received albendazole $50 \mathrm{mg} / \mathrm{Kg} /$ day for 2 years .Patient has been disease free since 2 years.

\section{Conclusion:-}

In nonendemic areas ecchinococcosis of head and neck region is very rare and missed and there fore it should be included in the differential diagnosis with a conscious descision.

\section{References:-}

[1]. Georgopaulos S etal. Hydatid cyst in the duct of the submandibular gland.Int.J Oral Maxillofacial Surgery2007;36:177-179.

[2]. Akhan etal. Percutaneous treatment of a parotid gland Hydatid cyst: a possible alternative to surgery. Eur Radiol 2002;12:597-599.

[3]. Lewis etal. A review of ecchinococcal disease.Ann Surg1995;18(4):390-396.

[4]. Primary hydatid cyst in the soft tissue of the face : An exceptional occurrence. Indian J Dermatology. 2011;56:768-770.

[5]. Ataoglu H etal.Maxillofacial hydatid cyst.J Oral Maxillofac Surg 2002;60:454-456.

[6]. Katilmis H etal Primary hydatid cyst of neck. Am J Otol Head and Neck Surg 2007;28:205-207.

[7]. Akyildiz AN etal. Hydatid cyst of the pterygopalatine fossa.j Oral Maxillofac Surg 1991; 49:87-89.

[8]. Umesh $\mathrm{K}$ et al. Hydatid cyst in infratemporal region-A rare case report.Al Ameen J Med Sci.Vol 3, No.1,2010.

[9]. Hydatid cyst in the maxillo facial region - sabri Shuker BDS J Oral an Maxillo facial surgery. Vol 40 issue 3; 171 -174 March 1982. 